Scientific Paper

\title{
Dosimetric comparison of deep inspiration breath hold and free breathing technique in stereotactic body radiotherapy for localized lung tumor using Flattening Filter Free beam
}

\author{
Karthick Raj MANI ${ }^{1,2, a}$, Md. Anisuzzaman BHUIYAN², Md. Mahbub ALAM³ ${ }^{3}$, Sharif AHMED², Mostafa Aziz SUMON², \\ Ashim Kumar SENGUPTA ${ }^{2}$, Md. Shakilur RAHMAN ${ }^{4}$, Md. S. M. Azharul ISLAM ${ }^{1}$ \\ ${ }^{1}$ Department of Physics, Jahangirnagar University, Savar, Dhaka - 1342, Bangladesh \\ ${ }^{2}$ Department of Radiation Oncology, United Hospital, Gulshan, Dhaka - 1212, Bangladesh \\ ${ }^{3}$ Department of Medical Physics and Biomedical Engineering, Gono Bishwabidyalay, Savar, Dhaka, Bangladesh \\ ${ }^{4}$ Secondary Standard Dosimetry Laboratory, Institute of Nuclear Science and Technology, Bangladesh Atomic Energy \\ Commission, Savar, Dhaka, Bangladesh \\ ${ }^{a}$ E-mail address: karthickrajmani@gmail.com
}

(received 12 October 2017; revised 18 December 2017; accepted 18 December 2017)

\begin{abstract}
Aim: To compare the dosimetric advantage of stereotactic body radiotherapy (SBRT) for localized lung tumor between deep inspiration breath hold technique and free breathing technique.

Materials and methods: We retrospectively included ten previously treated lung tumor patients in this dosimetric study. All the ten patients underwent CT simulation using 4D-CT free breathing (FB) and deep inspiration breath hold (DIBH) techniques. Plans were created using three coplanar full modulated arc using $6 \mathrm{MV}$ flattening filter free (FFF) bream with a dose rate of $1400 \mathrm{MU} / \mathrm{min}$. Same dose constraints for the target and the critical structures for a particular patient were used during the plan optimization process in DIBH and FB datasets. We intend to deliver 50 Gy in 5 fractions for all the patients. For standardization, all the plans were normalized at target mean of the planning target volume (PTV). Doses to the critical structures and targets were recorded from the dose volume histogram for evaluation.

Results: The mean right and left lung volumes were inflated by 1.55 and 1.60 times in DIBH scans compared to the FB scans. The mean internal target volume (ITV) increased in the FB datasets by 1.45 times compared to the DIBH data sets. The mean dose followed by standard deviation $\left(\overline{\mathrm{x}} \pm \sigma_{\overline{\mathrm{x}}}\right)$ of ipsilateral lung for DIBH-SBRT and FB-SBRT plans were $7.48 \pm 3.57(\mathrm{~Gy})$ and $10.23 \pm 4.58(\mathrm{~Gy})$ respectively, with a mean reduction of $36.84 \%$ in DIBH-SBRT plans. Ipsilateral lung were reduced to $36.84 \%$ in DIBH plans compared to FB plans.

Conclusion: Significant dose reduction in ipsilateral lung due to the lung inflation and target motion restriction in DIBH-SBRT plans were observed compare to FB-SBRT. DIBH-SBRT plans demonstrate superior dose reduction to the normal tissues and other critical structures.
\end{abstract}

Key words: DIBH; free breathing; SBRT; lung radiotherapy; gated SBRT.

\section{Introduction}

Organ motion management in radiotherapy has evolved in last few decades along with technology development. Stereotactic body radiation therapy (SBRT) requires precise delineation of patient anatomy, targets for planning, and clear visualization for localization during treatment delivery [1]. SBRT became a standard care of treatment for early stage lung cancer patients who are not fit for surgery [2]. In SBRT, precise delineation and localization of target is vital for an effective treatment. SBRT methodology is to deliver a highly conformal ablative dose to the tumor in a hypo-fractionation radiation schedule. SBRT requires biological equivalent dose (BED) $\geq 100$ Gy to achieve a tumor control of $85 \%$ irrespective of the tumor size in the primary and metastatic lung [3]. To safely practice SBRT, we need to follow stringent criteria starts from immobilization, delineation, treatment planning to treatment delivery. Motion management in moving organ is an important step in the SBRT treatment process, precious localization of the tumor during the target delineation and treatment delivery has to be in a high confidence level. To obtain the high confidence level, we need to have a clear idea about utilizing the available imaging technique to incorporate the tumor motion. International commission on Radiation Units and measurements (ICRU) report no 62 (Supplement to ICRU Report 50): Prescribing, Recording, and Reporting Photon Beam Therapy released in 1999 [4] recommended a term Internal Target Volume (ITV) in the moving tumors. ITV is 
described as a clinical target volume (CTV) with additional margin which encompass the tumor motion. Determination of accurate ITV become mandatory in SBRT; 4-dimensional computed tomography (4DCT) which is the combination of the 3D data of different respiratory phases enable us to determine the ITV. The maximum intensity projection (MIP) data set derived from all the respiratory phases become the standard golden data set for ITV delineation. There are different approach used by several authors Rosenzweig et al. [5] used deep inspiration breath hold (DIBH) technique where lung tumor can immobilized at a particular position and radiation field will be the smallest, hence using DIBH they can escalate the average from dose from $69.4 \mathrm{~Gy}$ to $87.9 \mathrm{~Gy}$, without increasing the risk of toxicity. In this dosimetric study we try to investigate the dosimetric advantages of DIBH-SBRT compared to the FB-SBRT.

\section{Materials and methods}

We retrospectively selected ten previously treated localized lung cancer patients stage (T1/T2, N0, M0) in this study. Out of the ten patients two patients were treated with deep inspiration breath hold technique and remaining eight patients treated with free breathing technique using 4D-CT. All the patients were underwent CT simulation with both 4dimensional Computed Tomography (4D-CT) and deep inspiration breath hold. The patient's demographic data were listed below in the Table 1.Ten patients with mean age of 54.7 years (range, 44 to 67 years) were retrospectively included in this study from our previously treated SBRT patient record. Out of ten patients six were right sided and four were left sided lung tumors. Among the ten patients six were male (four out of six were smokers) and four were female. The selected lung patients were mostly peripheral localized lung tumor, mostly had co-morbidities (i.e., COPD, Hypertension, etc.,) and not fit for surgery. The gender, age, stage and the location of the primary tumor along with the co-morbidities were listed in the Table 1.

\section{CT simulation}

All the patients were immobilized with custom made vacloc (M/S Civco, USA) fixed with ' $\mathrm{T}$ ' shaped wing board indexed to the couch. A computed tomography (CT) with a slice thickness of $2.5 \mathrm{~mm}$ was obtained for all the patients using GE Discovery 600 16-slice PET-CT scanner. Varian real time position management (RPM) equipped with infra-red camera and 6 dot reflective markers, which are integrated with the GE CT was used for all the patients to obtain the DIBH scans and 4D-CT scans. DIBH scans were acquired by helical mode, with $2.5 \mathrm{~mm}$ slice thickness, 16 rows detectors, $20 \mathrm{sec}$ breath hold, $400 \mathrm{~mm}$ field of view and without any inter slice gap. All the patients were evaluated for the compliance of ability to hold the breath for $20 \mathrm{sec}$. Prior to the DIBH scan, the patients were explained, coached, and assessed the upper and lower threshold cut off for all the patients. During the breath hold scan the upper and lower threshold cut off were set and documented.

During the simulation three sets of DIBH scans were taken for a patient to determine the ITV for the DIBH dataset. The 4DCT scans were acquired using a cine mode acquisition, with $2.5 \mathrm{~mm}$ slice thickness, 8 row detectors, $400 \mathrm{~mm}$ field of view, cine acquisition duration (breathing period of the individual patient $+0.5 \mathrm{sec}$ ), cine acquisition gap $0.45 \mathrm{sec}$ and without any inter slice gap. Once the cine acquisition completed the breath pattern with $\mathrm{CT}$ data acquisition information file was automatically transfer from the RPM system to the CT console. Using this file the cine acquisition data were divided into ten bins of respiratory phases (CT0, CT10, СT20, CT 30, CT40, CT50, CT60, CT70, CT80 and CT90). From the ten respiratory bins the MIP and the average intensity projection (AveIP) were also derived for all the patients. Figure 1 shows the DIBH and FB datasets, which clearly illustrates the lung inflation and reduced GTV volume. Once the patient CT data acquired, the CT images were imported into Eclipse treatment planning system, Ver. 11.0 (M/S Varian Medical Systems, Palo Alto, CA, USA). The body structure was segmented automatically by the treatment planning system.

Table 1. Patient demographic data

\begin{tabular}{ccccccc}
\hline \hline S No. & Stage & Age & Sex & Location & Co-Morbidities & $\begin{array}{c}\text { Treated } \\
\text { Technique }\end{array}$ \\
\hline 1 & T2aN0MO & 44 & M & Rt. Lower & Hypertension & FB \\
2 & T2bNOMO & 62 & F & Lt. Lower & COPD & FB \\
3 & T2aN0MO & 48 & M & Lt. Upper & Heart Disease & FB \\
4 & T2aN0MO & 53 & M & Lt. Lower & - & DIBH \\
5 & T1cN0MO & 65 & F & Rt. Upper & Hypertension & FB \\
6 & T2aN0MO & 49 & M & Rt. Lower & COPD & FB \\
7 & T1cN0MO & 51 & F & Lt. Upper & Heart Disease & FB \\
8 & T1bNOMO & 67 & M & Rt. Middle & - & DIBH \\
9 & T2aN0MO & 49 & M & Rt. Lower & Heart Disease & FB \\
10 & T2aN0MO & 59 & F & Rt. Upper & - & FB \\
\hline \hline
\end{tabular}

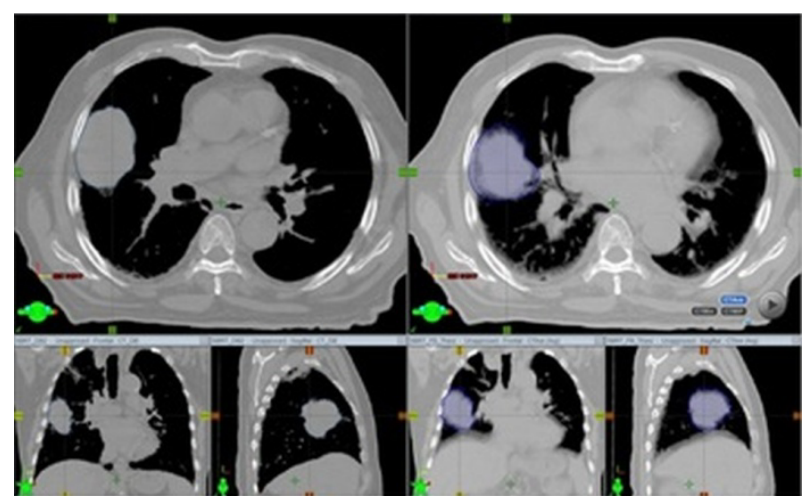

(a) (b)
Figure 1. Comparison of a) DIBH dataset and b) Free Breathing dataset 


\section{Critical structures contouring}

All the critical structures were contoured as per the RTOG 0915 report [6] guidelines. The spinal cord has been contoured in every slice, starting at least $10 \mathrm{~cm}$ above the superior extend of the PTV and finish at least $10 \mathrm{~cm}$ below the inferior extend of the PTV. Mediastinal window level was set on the planning CT to delineate the esophagus slice by slice, starting at least $10 \mathrm{~cm}$ above the superior extend of the PTV and finish at least $10 \mathrm{~cm}$ below the inferior extend of the PTV. The Heart was contoured beside the pericardial sac; the superior landmarks were the level of the inferior aspects of aortic arch and inferiorly extend up to the apex of the heart. Trachea and main bronchus which include the entire trachea, carina and proximal bronchial tree were delineated as a single structure. Both the right and left lungs has been contoured separately to estimate the dosimetric advantage in the ipsilateral lung between the DIBH and FB technique.

Contouring has been carried out using pulmonary windows. All inflated and collapsed lung has been included in this structures. The pulmonary trunk delineated from the anterior and medial wall of the right atrium, overlaps root of aorta and include the right and left pulmonary arteries. A 3D view of the all the critical structures and the target were illustrated in the Figure 2.

\section{Target delineation}

4DCT MIP datasets were used for the internal target volume delineation in the free breathing mode and they were verified by running the cine mode of the 10 phases CT data sets to ensure the target is inside the delineated ITV from the MIP data sets. We have acquired three DIBH data sets with common Dicom origin during the CT simulation process to include the uncertainty of the target within the upper and the lower threshold of the deep inspiration breath hold. The ITV was derived by delineating the target in all the three datasets which were shared a common Dicom origin as shown in the Figure 3. Both the DIBH and FB ITV's were expanded with 4 $\mathrm{mm}$ 3-dimensional margin to obtain the PTV.

\section{Treatment planning and optimization}

Eclipse treatment planning system (Varian Medical Systems, USA) was used for SBRT treatment planning for both DIBH and FB techniques. All the SBRT plans were planned with coplanar volumetric modulated arc therapy (VMAT) technique with 3 complete arcs. TrueBeam linear accelerator (Varian Medical Systems, USA) with 6 MV flattening filter free beam (FFF) using Millennium -120 multi-leaf collimator were used in all the plans. The DIBH-SBRT plans were done in DIBH CT datasets whereas FB-SBRT plans were done in the 4D-CT (AveIP) data sets. Progressive resolution optimization algorithm ver. 11.0 was used for the VMAT optimization. Dose calculation of both DIBH-SBRT and FB-SBRT plans were calculated using analytical anisotropic algorithm (AAA) ver.11.0. All the patients were planned to deliver $50 \mathrm{~Gy}$ in 5 fractions to the PTV. The dose constraints for a particular patient were kept identical during the optimization process for both DIBH-SBRT and FB-SBRT plan. To standardization the treatment plans, all the plans were normalized to the target mean of the PTV.

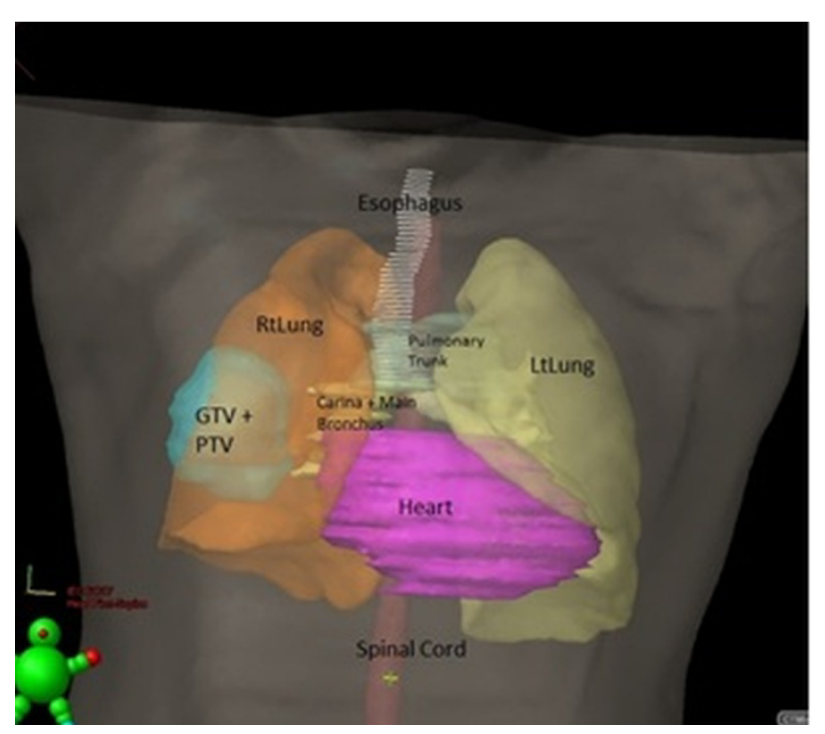

Figure 2. 3D-view of the critical structures and targets

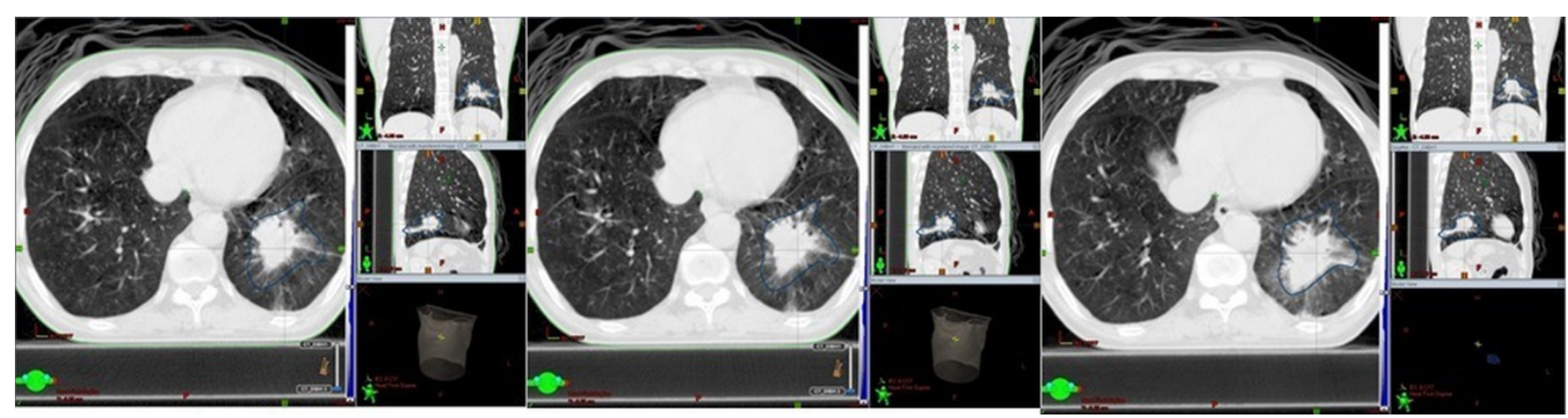

Figure 3. GTV variation in the DIBH dataset within the set upper and lower threshold done at three instances 


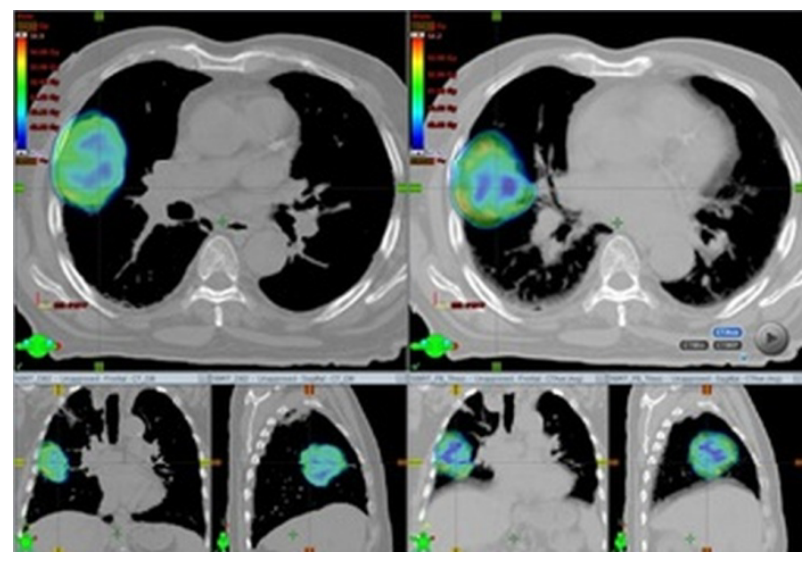

Figure 4. Dose distribution with a) DIBH technique and b) FB technique

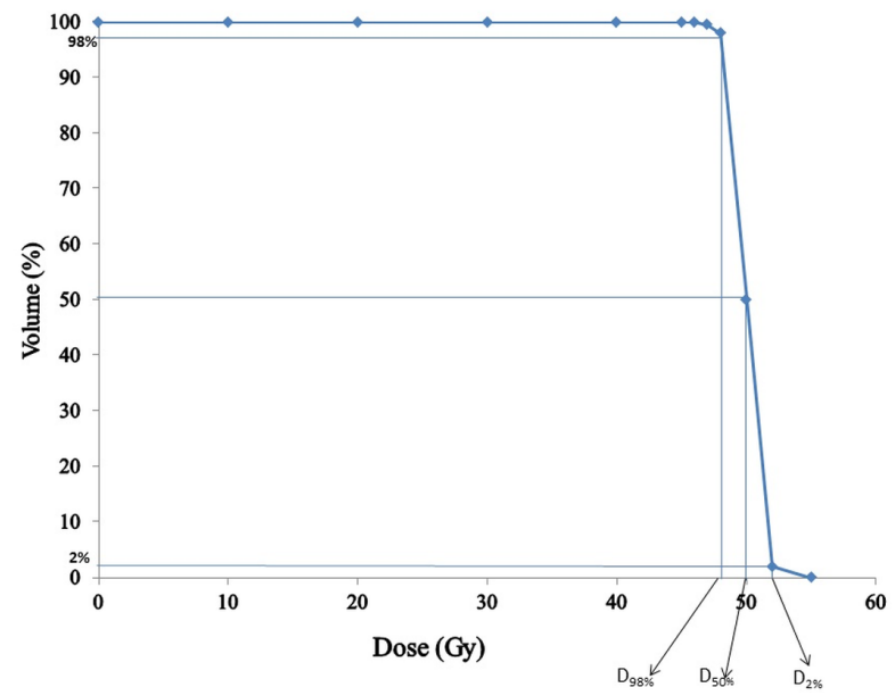

Figure 5. Determination of homogeneity index parameters from DVH

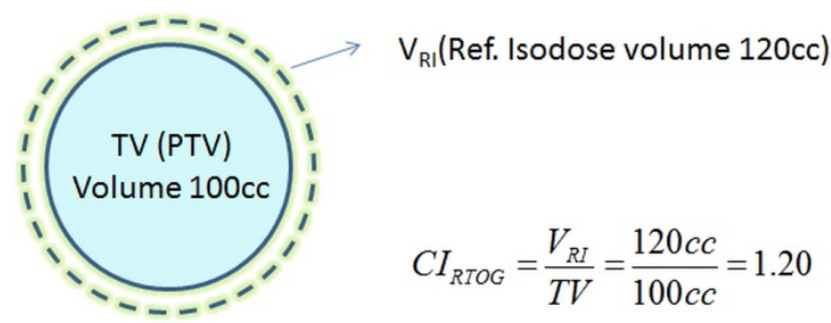

Figure 6. Example of Conformity Index

\section{Plan evaluation}

In the report ICRU report 83 [7], prescribing, recording and reporting photon beam intensity-modulated radiation therapy describes the dose homogeneity and dose conformity are independent specifications of the quality of the absorbed dose distribution. Dose homogeneity characterizes the uniformity of the absorbed-dose distribution within the target volume. Dose conformity characterizes the degree to which the high-dose region conforms to the target volume, usually the PTV.

\section{Homogeneity index (HI)}

$H I=\frac{D_{2 \%}-D_{98 \%}}{D_{50 \%}}$

Where, $\mathrm{D}_{2 \%}, \mathrm{D}_{98 \%}$, and $\mathrm{D}_{50 \%}$ are the dose received by $2 \%, 98 \%$ and $50 \%$ volumes respectively. $\mathrm{HI}=0$ (zero) is ideal value. The Figure 5 illustrates how to determine the $\mathrm{D}_{2 \%}, \mathrm{D}_{98 \%}$, and $\mathrm{D}_{50 \%}$ from the PTV in the dose volume histogram.

\section{Conformity index (CI)}

In 1993, Radiation Therapy Oncology Group recommended CI as a ratio of the reference isodose volume to the target volume.

$C I_{R T O G}=\frac{V_{R I}}{T V}$

Where, $V_{R I}$ reference isodose volume, and $T V$ is the target volume (i.e., PTV).

Figure 6 illustrates the example of computing the conformity index, for example if the PTV volume is $100 \mathrm{cc}$, where your reference isodose line (if the prescription is $50 \mathrm{~Gy}$, your reference isodose is $95 \%, 47.5$ Gy volume will be the reference isodose volume) is $120 \mathrm{cc}$, then the conformity index is calculated to be 1.20 , which describes there is $20 \%$ volume outside PTV receiving the prescribed dose.

\section{Patient specific quality assurance}

Patient specific quality assurances for DIBH-SBRT and FBSBRT plans (total 20 plans) were performed using I'mRT MatriXX 2-dimensional ion chamber array (Scanditronix Wellhofer, Freiburg, Germany). The fluence were measured using the I'mrt matrix attached to the linear accelerator head using the gantry mount with $5 \mathrm{~cm}$ of slab phantom above and below the detector to enable the buildup and backscatter radiation. With the same geometry we created the Eclipse TPS predicted fluence for all the patients. The TPS predicted fluence and the measured fluence from the machine were compared using the Omnipro IMRT software. All the 20 plans were compared between the TPS predicted and measured fluence using the gamma evaluation method with a acceptance criteria of $95 \%$ of the pixel within the region of interest has to pass $3 \%$ dose difference and $3 \mathrm{~mm}$ distance to agreement (DTA).

\section{Treatment localization}

All the DIBH-SBRT and FB-SBRT plans were added with three setup fields, two fields with orthogonal KV-KV images for 2D-2D bony match and the third field for the 3D-3D matching using CBCT. During the DIBH treatment localization, lower and upper threshold of the gated window was set as per the values documented during the CT 
simulation. DIBH gated $\mathrm{KV}-\mathrm{KV}$ image were acquired and match with the planning $\mathrm{CT}$ digitally reconstructed radiograph (DRR), by enabling the PTV outline. Verified that KV images tumour shadows were within the PTV outline in the DRR. (Figure 7).

The two patients treated with DIBH-SBRT target localization also verified using the breath hold gated CBCT option available with the TrueBeam Linear Accelerator. The breath hold gated CBCT gave us enough confident about the accuracy of the tumor immobilized in the set upper and lower threshold by visualizing the tumor within the planning PTV as shown in the Figure 8.

\section{Statistical analysis:}

All the statistical data were presented in study as the mean followed by the standard deviation $\left(\overline{\mathrm{x}} \pm \sigma_{\overline{\mathrm{x}}}\right)$. The DIBH-SBRT plans dosimetric data were compared with the FB-SBRT plans data using the paired sample t-test performed using the Microsoft Excel version 2010 with p value $<0.05$ considered as significant.

\section{Results and Discussion}

\section{ITV and PTV volume}

The mean followed by standard deviation $\left(\overline{\mathrm{x}} \pm \sigma_{\overline{\mathrm{x}}}\right)$ of DIBHITV and DIBH - PTV volume (cc) of all the patients were $51.29 \pm 47.13$ (range: $5.40-131.70$ ) and $92.01 \pm 76.62$ (range: 15.60 - 246.30), whereas FB -ITV and FB - PTV volume were $74.42 \pm 69.06$ (range: $6.40-211.50$ ) and $112.66 \pm 94.20$ (range: $18.50-323.30$ ). We found that mean FB-ITV of all the patients were 1.45 times of DIBH-ITV and FB-PTV were 1.22 times larger than the DIBH-PTV. The volume variation of ITV and PTV between the two techniques were illustrated in the Figure 9.The reductions of the ITV in the DIBH datasets were mainly due to immobilize the target within the gated upper and lower threshold window.

\section{Conformity index and homogeneity index for PTV}

The treatment plan quality of the DIBH-SBRT and FB-SBRT techniques were compared using the dose homogeneity index (HI) and dose conformity index (CI) using the Equation 1 and 2. The calculated dose homogeneity and conformity index between the two techniques were listed in Table 2. The mean followed by standard deviation $\left(\overline{\mathrm{x}} \pm \sigma_{\overline{\mathrm{x}}}\right)$ of CI in the DIBHSBRT and FB-SBRT plans were $1.22 \pm 0.09$ and $1.21 \pm 0.09$, $\mathrm{HI}$ for the the DIBH-SBRT and FB-SBRT plans were $0.17 \pm$ 0.03 and $0.13 \pm 0.02$.

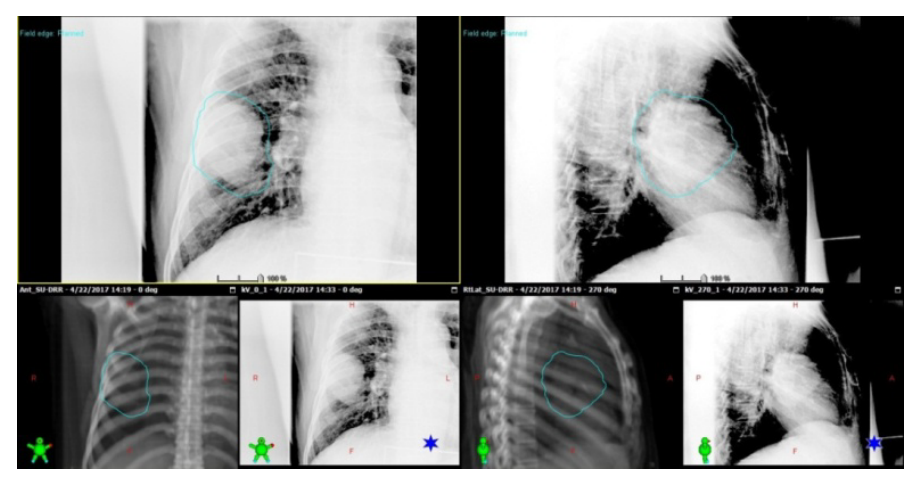

Figure 7. 2D-2D gated DIBH verification using orthogonal images

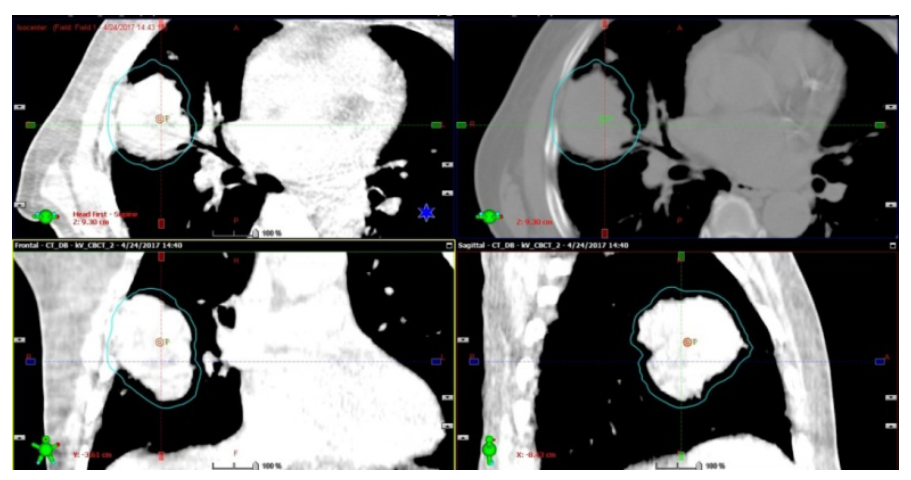

Figure 8. 3D-3D gated DIBH CBCT verification

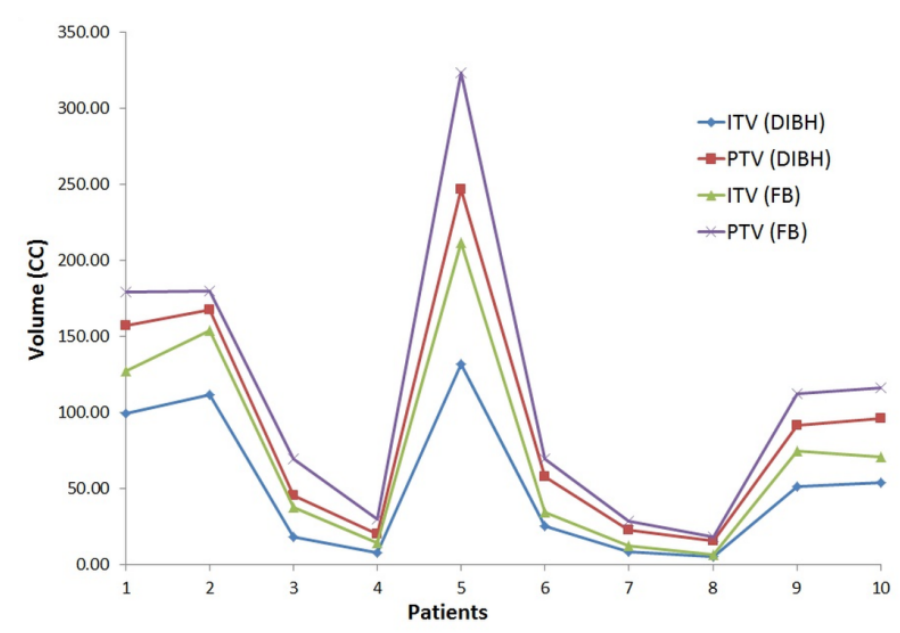

Figure 9: DIBH versus FB (ITV \& PTV)

Table 2. Conformity and homogeneity index for DIBH-SBRT and FB-SBRT

\begin{tabular}{ccc}
\hline \hline Parameter & DIBH $\left(\overline{\mathrm{x}} \pm \sigma_{\overline{\mathrm{x}}}\right)$ & FB $\left(\overline{\mathrm{x}} \pm \sigma_{\overline{\mathrm{x}}}\right)$ \\
\hline $\mathrm{D}_{2 \%}(\mathrm{~Gy})$ & $53.25 \pm 0.61$ & $52.48 \pm 0.38$ \\
$\mathrm{D}_{50 \%}(\mathrm{~Gy})$ & $50.10 \pm 0.17$ & $50.13 \pm 0.20$ \\
$\mathrm{D}_{98 \%}(\mathrm{~Gy})$ & $44.96 \pm 1.02$ & $45.95 \pm 0.89$ \\
95\% isodose vol. (cc) & $109.02 \pm 103.07$ & $125.99 \pm 120.54$ \\
PTV vol. (cc) & $91.55 \pm 86.87$ & $107.98 \pm 106.21$ \\
$\mathrm{CI}_{\text {RTOG }}$ & $1.22 \pm 0.09$ & $1.21 \pm 0.09$ \\
HI & $0.17 \pm 0.03$ & $0.13 \pm 0.02$ \\
\hline \hline
\end{tabular}




\section{Normal tissue (Body)}

The dose comparison of the whole body low dose and high dose volumes for the DIBH-SBRT and FB-SBRT were listed in the Table 3. The DIBH-SBRT plans shows significant dose reduction in the low dose as well as high dose volumes compared to the FB-SBRT technique. The $\overline{\mathrm{x}} \pm \sigma_{\overline{\mathrm{x}}}$ of $\mathrm{V}_{5}, \mathrm{~V}_{10}$, $\mathrm{V}_{20}, \mathrm{~V}_{30}, \mathrm{~V}_{40}$ and $\mathrm{V}_{50}$ represented in percentage of volume $(\%)$ for the DIBH-SBRT were $12.37 \pm 5.17,6.01 \pm 3.47,2.31 \pm$ $1.83,1.19 \pm 0.98,0.71 \pm 0.59$ and $0.26 \pm 0.22$ respectively. The $\overline{\mathrm{x}} \pm \sigma_{\overline{\mathrm{x}}}$ of $\mathrm{V}_{5}, \mathrm{~V}_{10}, \mathrm{~V}_{20}, \mathrm{~V}_{30}, \mathrm{~V}_{40}$ and $\mathrm{V}_{50}$ represent in percentage of volume $(\%)$ for the FB-SBRT were $13.10 \pm 5.73,6.67 \pm$ $3.62,2.62 \pm 1.12,1.39 \pm 1.12,0.87 \pm 0.72$ and $0.33 \pm 0.28$ respectively. The DIBH-SBRT shows significant dose reduction in $\mathrm{V}_{10}, \mathrm{~V}_{20}, \mathrm{~V}_{30}, \mathrm{~V}_{40}$ and $\mathrm{V}_{50}$ which are statistically significant, with a mean dose reduction of $7.96 \%$ in the normal tissue compared to the FB-SBRT. The dose volume histogram comparison of normal tissue (Body) for a patient between DIBH-SBRT and FB-SBRT is shown in the Figure 10.

\section{Ipsilateral Lung}

The dosimetric comparison parameters of the ipsilateral lung were tabulated in the Table 3. The $\bar{x} \pm \sigma_{\bar{x}}$ of $V_{5}, V_{10}, V_{20}, V_{30}$, $\mathrm{V}_{40}$ and $\mathrm{V}_{50}$ represented in percentage of volume $(\%)$ for the DIBH-SBRT were $33.40 \pm 11.88,25.31 \pm 11.67,12.90 \pm 9.08$, $7.28 \pm 6.22,4.22 \pm 4.01$ and $1.33 \pm 1.45$ respectively. The percentage mean \pm standard deviation $\left(\overline{\mathrm{x}} \pm \sigma_{\overline{\mathrm{x}}}\right)$ of $\mathrm{V}_{5}, \mathrm{~V}_{10}, \mathrm{~V}_{20}$, $\mathrm{V}_{30}, \mathrm{~V}_{40}$ and $\mathrm{V}_{50}$ for the FB-SBRT were $40.94 \pm 15.38,33.34 \pm$ $15.01,18.85 \pm 10.88,11.60 \pm 7.42,7.43 \pm 5.30$ and $2.68 \pm 2.28$ respectively. The dose volume comparison of the ipsilateral lung between the DIBH-SBRT and FB-SBRT technique is shown in the Figure 11. The $\overline{\mathrm{x}} \pm \sigma_{\overline{\mathrm{x}}}$ of ipsilateral lung mean dose for DIBH-SBRT and FB-SBRT plans were 7.48 \pm 3.57 and $10.23 \pm 4.58$ respectively, with a mean reduction of $36.84 \%$ in the DIBH-SBRT and they were highly statistical significant ( $\mathrm{p}$ value 0.00 ).

\section{Contralateral Lung}

We have not observed any high dose volume in the contralateral lung. The dosimetric comparisons of both the techniques for the contralateral lung were listed in the Table 3. The $\overline{\mathrm{x}} \pm \sigma_{\overline{\mathrm{x}}}$ represented in percentage of volume $(\%)$ for DIBHSBRT plans were $\mathrm{V}_{5}(17.27 \pm 10.36)$ and $\mathrm{V}_{10}(1.29 \pm 0.79)$ and whereas for FB-SBRT plans were $\mathrm{V}_{5}(19.47 \pm 14.87)$ and $\mathrm{V}_{10}$ $(2.31 \pm 1.31)$.The DIBH-SBRT plans show a mean reduction of $12.73 \%$ in $\mathrm{V}_{5}$ and $78.75 \%$ in $\mathrm{V}_{10}$ compared to the FB-SBRT plans. The mean $\left(\overline{\mathrm{x}} \pm \sigma_{\overline{\mathrm{x}}}\right)$ dose (Gy) of the contralateral lung for the DIBH-SBRT and FB-SBRT were $2.09 \pm 0.89$ and $2.41 \pm$ 1.14 with a mean reduction of $15.21 \%$ and they were statistical significant ( $\mathrm{p}$ value 0.08). The dose volume histogram comparison of contralateral lung for a patient between DIBHSBRT and FB-SBRT plans is shown in the Figure 11.

\section{Esophagus}

Since all the patients included in this study were peripheral tumors, we have not observed any high dose volume in the esophagus. The dosimetric comparisons of both the techniques for the esophagus were listed in the Table 3. We found $\bar{x} \pm \sigma_{\bar{x}}$ represented in percentage (\%) of volume for DIBH-SBRT plans were $\mathrm{V}_{5}(26.34 \pm 12.80)$ and $\mathrm{V}_{10}(14.20 \pm 7.94)$, whereas FB-SBRT plans resulted in $\mathrm{V}_{5}(31.21 \pm 10.35)$ and $\mathrm{V}_{10}(18.46 \pm$ 9.21). The DIBH-SBRT plans show a mean reduction of $15.60 \%$ in $\mathrm{V}_{5}$ and $23.06 \%$ in $\mathrm{V}_{10}$ compared to the FB-SBRT. The mean dose of esophagus for the DIBH-SBRT and FBSBRT were $3.15 \pm 1.39$ and $4.04 \pm 1.45$ (Gy) with a mean reduction of $22.02 \%$ and they were highly statistical significant ( $\mathrm{p}$ value 0.001 ). The dose volume histogram comparison of esophagus for a patient between DIBH-SBRT and FB-SBRT plan is shown in the Figure 11.

\section{Pulmonary Trunk}

As the pulmonary trunk is a midline structure the high dose volumes were not found, only the low dose volumes $\mathrm{V}_{5}, \mathrm{~V}_{10}$, $\mathrm{V}_{20}, \mathrm{~V}_{30}$ and the mean dose (Gy) were listed for the DIBHSBRT and FB-SBRT in the Table 3. The $\bar{x} \pm \sigma_{\bar{x}}$ of the pulmonary trunk were $\mathrm{V}_{5}(30.54 \pm 12.12), \mathrm{V}_{10}(20.59 \pm 12.52)$, $\mathrm{V}_{20}(2.06 \pm 1.23), \mathrm{V}_{30}(0.56 \pm 0.32)$ and mean dose $(4.31 \pm$ 2.78) for the DIBH-SBRT, whereas for FB-SBRT the $V_{5}$ $(49.76 \pm 33.56), V_{10}(26.64 \pm 16.69), V_{20}(5.35 \pm 3.56), V_{30}$ $(1.21 \pm 0.68)$ and mean dose $(6.57 \pm 4.75)$. The DIBH-SBRT plans show a mean reduction of $38.62 \%$ in $\mathrm{V}_{5}, 22.71 \%$, in $\mathrm{V}_{10}$, $61.55 \%$ in $\mathrm{V}_{20}, 53.79 \%$ in $\mathrm{V}_{30}$ and $34.38 \%$ in mean dose compared to the FB-SBRT. The dose volume histogram comparison of pulmonary trunk for a patient between DIBHSBRT and FB-SBRT plan is shown in the Figure 11.

\section{Heart}

The dosimetric parameters between the DIBH-SBRT and FBSBRT plans were listed in the Table 3. The $\bar{x} \pm \sigma_{\bar{x}}$ represented in the dose (Gy) for the heart in DIBH-SBRT $(4.23 \pm 2.89)$ and FB-SBRT $(4.81 \pm 3.20)$, with a mean reduction of $11.98 \%$ in DIBH-SBRT compare to the FB-SBRT plans. The $\overline{\mathrm{x}} \pm \sigma_{\overline{\mathrm{x}}}$ of heart in DIBH-SBRT plans represented in the percentage (\%) of volume were $\mathrm{V}_{5}(31.76 \pm 21.09), \mathrm{V}_{10}(13.87 \pm 15.97), \mathrm{V}_{20}$ $(2.08 \pm 1.37)$ and $\mathrm{V}_{30}(0.32 \pm 0.21)$, whereas FB-SBRT plans resulted in $\mathrm{V}_{5}(33.05 \pm 28.52), \mathrm{V}_{10}(16.81 \pm 12.52), \mathrm{V}_{20}(2.90 \pm$ $1.85)$ and $\mathrm{V}_{30}(0.80 \pm 0.46)$ for the. We found the mean heart doses of DIBH-SBRT plans were significantly less compared to the FB-SBRT plans with a statistical significance (p-value 0.017). The dose volume comparison of the heart of DIBHSBRT and FB-SBRT were shown in the Figure 12. 
Table 3. Dose comparison and statistical significance of low dose volume and OARs between DIBH and FB

\begin{tabular}{|c|c|c|c|c|}
\hline & $\overline{\text { DIBH }\left(\bar{x} \pm \sigma_{\bar{x}}\right)}$ & $\overline{\text { FB }\left(\bar{x} \pm \sigma_{\bar{x}}\right)}$ & Difference (\%) & p - value \\
\hline \multicolumn{5}{|c|}{ Body } \\
\hline Volume (cc) & $19363.05 \pm 3296.86$ & $19060.13 \pm 3624.04$ & & \\
\hline V5 (\%) & $12.37 \pm 5.17$ & $13.10 \pm 5.73$ & $5.53 \%$ & 0.200 \\
\hline $\mathrm{V} 10(\%)$ & $6.01 \pm 3.47$ & $6.67 \pm 3.62$ & $9.96 \%$ & 0.022 \\
\hline $\mathrm{V} 20(\%)$ & $2.31 \pm 1.83$ & $2.62 \pm 1.12$ & $11.91 \%$ & 0.003 \\
\hline $\mathrm{V} 30(\%)$ & $1.19 \pm 0.98$ & $1.39 \pm 1.12$ & $14.72 \%$ & 0.017 \\
\hline V40 (\%) & $0.71 \pm 0.59$ & $0.87 \pm 0.72$ & $17.71 \%$ & 0.024 \\
\hline $\mathrm{V} 50(\%)$ & $0.26 \pm 0.22$ & $0.33 \pm 0.28$ & $19.84 \%$ & 0.029 \\
\hline Mean (Gy) & $2.19 \pm 1.04$ & $2.34 \pm 1.13$ & $7.96 \%$ & 0.026 \\
\hline \multicolumn{5}{|c|}{ Ipsilateral Lung } \\
\hline Volume(cc) & $1875.90 \pm 474.15$ & $1198.24 \pm 371.62$ & & \\
\hline V5 (\%) & $33.40 \pm 11.88$ & $40.94 \pm 15.38$ & $22.59 \%$ & 0.01 \\
\hline V10 $(\%)$ & $25.31 \pm 11.67$ & $33.34 \pm 15.01$ & $31.73 \%$ & 0.00 \\
\hline $\mathrm{V} 20(\%)$ & $12.90 \pm 9.08$ & $18.85 \pm 10.88$ & $46.21 \%$ & 0.00 \\
\hline $\mathrm{V} 30(\%)$ & $7.28 \pm 6.22$ & $11.60 \pm 7.42$ & $59.45 \%$ & 0.01 \\
\hline $\mathrm{V} 40(\%)$ & $4.22 \pm 4.01$ & $7.43 \pm 5.30$ & $76.12 \%$ & 0.01 \\
\hline V50 (\%) & $1.33 \pm 1.45$ & $2.68 \pm 2.28$ & $100.48 \%$ & 0.03 \\
\hline Mean (Gy) & $7.48 \pm 3.57$ & $10.23 \pm 4.58$ & $36.84 \%$ & 0.00 \\
\hline \multicolumn{5}{|c|}{ Contralateral Lung } \\
\hline Volume (cc) & $1856.58 \pm 492.62$ & $1168 \pm 351.21$ & & \\
\hline V5 (\%) & $17.27 \pm 10.36$ & $19.47 \pm 14.87$ & $12.73 \%$ & 0.27 \\
\hline $\mathrm{V} 10(\%)$ & $1.29 \pm 0.79$ & $2.31 \pm 1.31$ & $78.75 \%$ & 0.26 \\
\hline Mean (Gy) & $2.09 \pm 0.89$ & $2.41 \pm 1.14$ & $15.21 \%$ & 0.08 \\
\hline \multicolumn{5}{|c|}{ Heart } \\
\hline Volume (cc) & $442.64 \pm 120.40$ & $481.06 \pm 123.21$ & & \\
\hline V5 (\%) & $31.76 \pm 21.09$ & $33.05 \pm 28.52$ & $3.89 \%$ & 0.785 \\
\hline $\mathrm{V} 10(\%)$ & $13.87 \pm 15.97$ & $16.81 \pm 12.52$ & $17.49 \%$ & 0.129 \\
\hline $\mathrm{V} 20(\%)$ & $2.08 \pm 1.37$ & $2.90 \pm 1.85$ & $28.31 \%$ & 0.276 \\
\hline $\mathrm{V} 30(\%)$ & $0.32 \pm 0.21$ & $0.80 \pm 0.46$ & $59.22 \%$ & 0.242 \\
\hline Mean (Gy) & $4.23 \pm 2.89$ & $4.81 \pm 3.20$ & $11.98 \%$ & 0.017 \\
\hline \multicolumn{5}{|c|}{ Esophagus } \\
\hline Volume (cc) & $36.96 \pm 6.31$ & $38.23 \pm 6.87$ & & \\
\hline V5 (\%) & $26.34 \pm 12.80$ & $31.21 \pm 10.35$ & $15.60 \%$ & 0.034 \\
\hline $\mathrm{V} 10(\%)$ & $14.20 \pm 7.94$ & $18.46 \pm 9.21$ & $23.06 \%$ & 0.005 \\
\hline Mean (Gy) & $3.15 \pm 1.39$ & $4.04 \pm 1.45$ & $22.02 \%$ & 0.001 \\
\hline \multicolumn{5}{|c|}{ Spinal Cord } \\
\hline Volume (cc) & $23.91 \pm 4.79$ & $24.46 \pm 6.30$ & & \\
\hline V5 (\%) & $15.86 \pm 6.00$ & $18.23 \pm 7.44$ & $13.01 \%$ & 0.059 \\
\hline V10 (\%) & $6.00 \pm 4.44$ & $6.64 \pm 4.85$ & $9.66 \%$ & 0.273 \\
\hline Mean (Gy) & $1.90 \pm 0.80$ & $2.21 \pm 1.01$ & $13.73 \%$ & 0.023 \\
\hline Max. (Gy) & $13.20 \pm 3.85$ & $13.91 \pm 4.44$ & $5.10 \%$ & 0.031 \\
\hline \multicolumn{5}{|c|}{ Pulmonary Trunk } \\
\hline Volume (cc) & $35.85 \pm 13.85$ & $34.74 \pm 16.19$ & & \\
\hline V5 (\%) & $30.54 \pm 12.12$ & $49.76 \pm 33.56$ & $38.62 \%$ & 0.052 \\
\hline $\mathrm{V} 10(\%)$ & $20.59 \pm 12.52$ & $26.64 \pm 16.69$ & $22.71 \%$ & 0.05 \\
\hline V20 (\%) & $2.06 \pm 1.23$ & $5.35 \pm 3.56$ & $61.55 \%$ & 0.298 \\
\hline V30 (\%) & $0.56 \pm 0.32$ & $1.21 \pm 0.68$ & $53.79 \%$ & 0.342 \\
\hline Mean (Gy) & $4.31 \pm 2.78$ & $6.57 \pm 4.75$ & $34.38 \%$ & 0.013 \\
\hline \multicolumn{5}{|c|}{ Main Bronchus + Trachea } \\
\hline Volume (cc) & $36.25 \pm 13.56$ & $34.49 \pm 16.30$ & & \\
\hline V5 (\%) & $41.39 \pm 26.72$ & $44.15 \pm 31.92$ & $6.24 \%$ & 0.573 \\
\hline $\mathrm{V} 10(\%)$ & $23.69 \pm 15.69$ & $28.44 \pm 27.10$ & $16.68 \%$ & 0.041 \\
\hline $\mathrm{V} 20(\%)$ & $3.63 \pm 2.75$ & $4.57 \pm 3.75$ & $20.68 \%$ & 0.993 \\
\hline V30 (\%) & $0.33 \pm 0.21$ & $0.45 \pm 0.23$ & $27.32 \%$ & 0.609 \\
\hline Mean (Gy) & $5.72 \pm 3.66$ & $6.65 \pm 3.95$ & $13.98 \%$ & 0.004 \\
\hline
\end{tabular}

Abbreviations: DIBH - Deep Inspiration Breath Hold, FB - Free Breathing, V5 (\%), V10 (\%),V20 (\%),V30 (\%),V40 (\%) and V50 (\%) are the percentage of volume receiving at-least $5 \mathrm{~Gy}, 10 \mathrm{~Gy}, 20 \mathrm{~Gy}, 30 \mathrm{~Gy}, 40 \mathrm{~Gy}$ and 50 Gy respectively. 


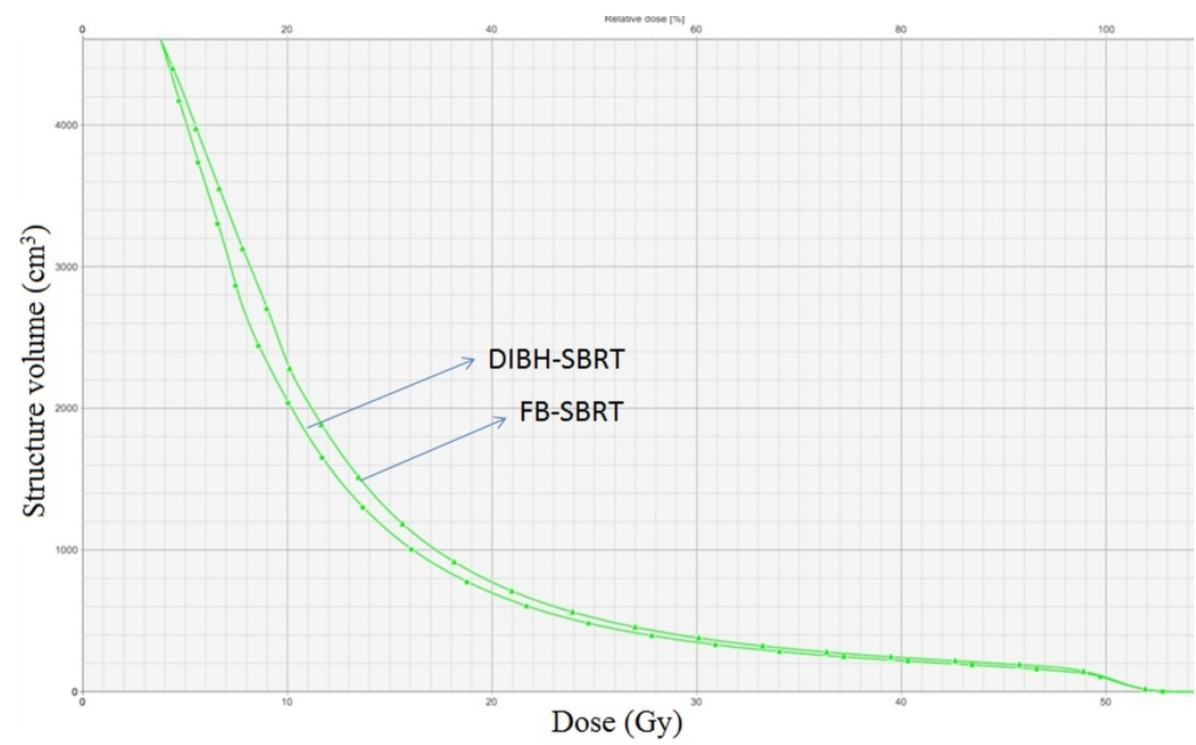

Figure 10. Normal tissue low dose volume (DIBH versus FB)

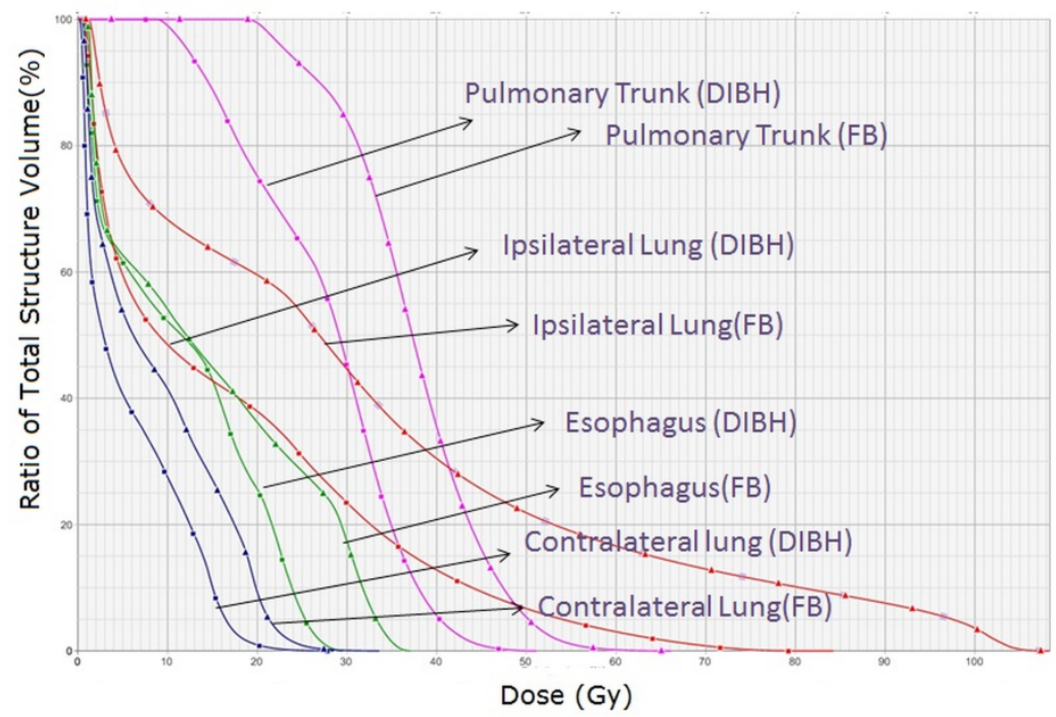

Figure 11. OAR's DVH comparisons (DIBH versus FB)

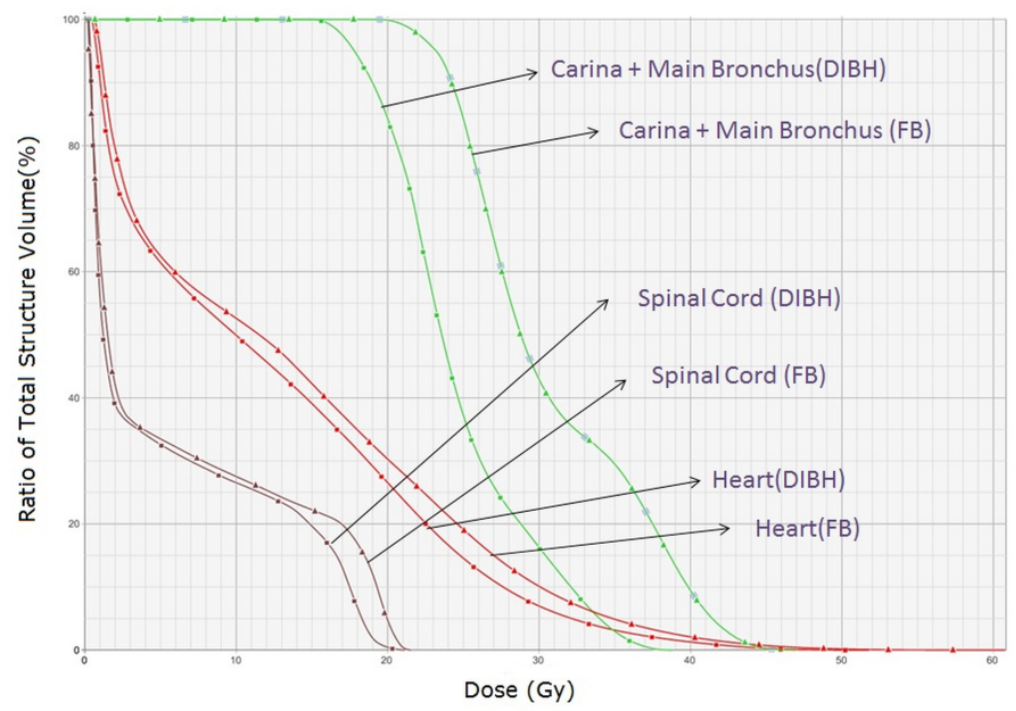

Figure 12. OAR's DVH comparisons (DIBH versus FB) 


\section{Main bronchus + Carina}

The low dose volumes $\mathrm{V}_{5}, \mathrm{~V}_{10}, \mathrm{~V}_{20}, \mathrm{~V}_{30}$ and the mean dose (Gy) of the main bronchus + carina were listed for the DIBH-SBRT and FB-SBRT in the Table 3. The $\bar{x} \pm \sigma_{\bar{x}}$ of the main bronchus + carina were $\mathrm{V}_{5}(41.39 \pm 26.72), \mathrm{V}_{10}(23.69 \pm 15.69), \mathrm{V}_{20}$ $(3.63 \pm 2.75), \mathrm{V}_{30}(0.33 \pm 0.21)$ and mean dose $(5.72 \pm 3.66)$ for the DIBH-SBRT, whereas for FB-SBRT the $\mathrm{V}_{5}(44.15 \pm$ $31.92), \mathrm{V}_{10}(28.44 \pm 27.10), \mathrm{V}_{20}(4.57 \pm 3.75), \mathrm{V}_{30}(0.45 \pm$ $0.23)$ and mean dose $(6.65 \pm 3.95)$. The DIBH-SBRT plans show a mean reduction of $6.24 \%$ in $\mathrm{V}_{5}, 16.68 \%$, in $\mathrm{V}_{10}$, $20.68 \%$ in $\mathrm{V}_{20}, 27.32 \%$ in $\mathrm{V}_{30}$ and $13.98 \%$ in mean dose compared to the FB-SBRT. The dose volume histogram comparison of main bronchus + carina for a patient between DIBH-SBRT and FB-SBRT is shown in the Figure 12.

\section{Spinal cord}

The dosimetric parameters $\mathrm{V}_{5}, \mathrm{~V}_{10}$, mean dose and maximum doses between the DIBH-SBRT and FB-SBRT were listed in the Table 3. The maximum dose to the spinal cord $\left(\overline{\mathrm{x}} \pm \sigma_{\overline{\mathrm{x}}}\right)$ represented in dose (Gy) were DIBH-SBRT (13.20 \pm 3.85$)$ and FB-SBRT $(13.91 \pm 4.44)$, which shows a mean reduction of $5.1 \%$ in DIBH-SBRT compare to the FB-SBRT with a statistical significance (p-value 0.031). The $\overline{\mathrm{x}} \pm \sigma_{\overline{\mathrm{x}}}$ of DIBHSBRT plans were $\mathrm{V}_{5}(15.86 \pm 6.00), \mathrm{V}_{10}(6.00 \pm 6.44)$ and mean dose $(1.90 \pm 0.80)$ for the DIBH-SBRT and $\mathrm{V}_{5}(18.23 \pm$ $7.44), V_{10}(6.64 \pm 4.85)$ and mean dose $(2.21 \pm 1.01)$ for the FB-SBRT. The dose volume comparison of the spinal cord of DIBH-SBRT and FB-SBRT plan were shown in the Figure 12.

\section{Patient specific quality assurance results}

Patient specific quality assurance for all the DIBH-SBRT and FB-SBRT plans (total 20 plans) were performed using I'mRT MatriXX 2-dimensional ion chamber (Scanditronix Wellhofer, Freiburg, Germany). The treatment planning system predicted fluence and the machine delivered fluence were evaluated using gamma evaluation criteria of 3mm DTA and 3\% delta dose difference. All the twenty plans passed the criteria, the mean percentage $\left(\overline{\mathrm{x}} \pm \sigma_{\overline{\mathrm{x}}}\right)$ pixel passed in DIBH-SBRT plans were $97.3 \pm 1.65$, whereas FB-SBRT plans were $97.7 \pm 1.82$. This result concludes that TPS predicted fluence and the machine delivered fluence for all the twenty plans are within the acceptable tolerance.

\section{Discussion}

Two of ten patients received DIBH-SBRT comfortably during the treatment course; the gated DIBH CBCT shows great confidence in the tumor localization during the treatment delivery. The DIBH mean followed by standard deviation $(\overline{\mathrm{x}} \pm$ $\left.\sigma_{\overline{\mathrm{x}}}\right)$ of the right lung and left lung volume (cc) were $1939.85 \pm$ $492.411792 .63 \pm 461.72$ compare to the FB mean volume of $1248.60 \pm 365.32$ and $1117.96 \pm 344.97$ respectively. In the DIBH datasets the mean right lung and the left lung volumes has been increased due to the inflation by 1.55 (range: 1.39 1.86) and 1.60 (range: $1.40-1.99$ ) times more than the FB. Josipovic et al. [8] has also compared the DIBH datasets with FB data sets of ten patients and found that in DIBH, the lung volume increase substantially for all patients, by mean of $57 \%$ (range $35 \%$ to $85 \%$ ), which is similar with our results.

The mean DIBH-ITV and DIBH-PTV volume (cc) of all the patients $\left(\overline{\mathrm{x}} \pm \sigma_{\overline{\mathrm{x}}}\right)$ were $51.29 \pm 47.13$ (range: $5.40-131.70$ ) and $92.01 \pm 76.62$ (range: $15.60-246.30$ ), whereas the FB were $74.42 \pm 69.06$ (range: $6.40-211.50$ ) and $112.66 \pm 94.20$ (range: $18.50-323.30$ ) respectively. We found that mean FBITV of all the patients were 1.45 times of DIBH-ITV and FBPTV were 1.22 times larger than the DIBH-PTV. Giraud et al [9], conducted a non-standardized multicenter study which includes 401 patients comparing the DIBH and FB datasets, which resulted in the PTV volume (cc) of the DIBH was $282 \pm$ 176 and FB was $360 \pm 232$ respectively (p value 0.00001 ). This study shows that the DIBH-PTV was 1.28 times larger than FB-PTV, which is comparable to our study.

We found that the increase in the PTV volume in the FBSBRT compare to the DIBH-SBRT, FB-SBRT resulted in the higher dose to the lung and the critical structures. In DIBHSBRT the mean heart dose was reduced by $11.98 \%$ and V20 by $28.31 \%$ (both the mean dose and V20 shows statistical significance) compare to the FB-SBRT. Ipsilateral lung for the DIBH-SBRT plans shows a mean reduction of $36.84 \%$ in mean dose, $46.21 \%$ in V20 and $76.12 \%$ in the V40 compare to the FB-SBRT plans. In this study we have not removed the PTV from the ipsilateral lung volume, hence compare to the other studies higher mean dose were documented.

The mean conformity index and the homogeneity index of the both DIBH-SBRT and FB-SBRT plans were comparable, which shows both the techniques on target dose confirmation and dose homogeneity were similar.

All the patients included in this study were peripheral lung tumors; hence the doses to the middle critical structures were comparatively less. The mean doses of esophagus, pulmonary trunk and main bronchus + carina in DIBH-SBRT were $22.02 \%, 34.38 \%$ and $13.98 \%$ less compare to the FB-SBRT plans.

DIBH CT images characterizes a perfect absence of motion artifacts due to the nature of the breath hold CT data acquisition, which resulted in superior target boundary definition compare to the FB scans. To practice DIBH based SBRT, target re-localization during the treatment is an important component. In this study we had verified the relocalization during the treatment by gated DIBH KV $-\mathrm{KV}$ imaging with the overlay of the PTV in the DRR and a gated DIBH CBCT to verify the target position. Gated DIBH CBCT provides enough confidence about the DIBH-SBRT technique delivery accuracy; hence to practice DIBH-SBRT we need a strong image guidance tool to validate the treatment relocalization of the target. 
We found that there is considerable amount of tumor motion within the upper and lower threshold of the DIBH scans; hence it is advisable to perform at-least three DIBH scans during the CT simulation with the same Dicom origin and Boolean the target volume to form an ITV. This will avoid a lot of residual error in re-localization during the treatment delivery.

In this work, we have treated two patients using DIBH based SBRT and the compliance of both the patients from the CTsimulation, treatment position verification to treatment delivery completion was satisfactory. We need to be careful in the patient selection and patient's compliance to proceed with DIBH based SBRT.

\section{Conclusion}

DIBH-SBRT plans are much superior due to smaller PTV due to its capability of immobilizing the target motion during the treatment within the threshold window. In this study we also found that the DIBH based SBRT significantly reduces the doses to the ipsilateral lung due to lung inflation, which will results in less lung toxicity compare to the FB based SBRT. We need well equipped advanced CT simulator with gating system and image guidance tool to practice safe DIBH based SBRT treatment.

\section{References}

[1] Benedict SH, Yenice KM, Followill D, et. al Stereotactic body radiation therapy: The report of AAPM Task Group 101. Med Phys. 2010;37:4078-4101.

[2] Timmerman R, Paulus R, Galvin J, et al.Stereotactic body radiation therapy (SBRT) to treat medically inoperable early stage lung cancer patients: Analysis of RTOG 0236. Int J Radiat Oncol Biol Phys. 2009;75(3):S3.

[3] Park S, Urm S, Cho H. Analysis of Biologically Equivalent Dose of Stereotactic Body Radiotherapy for Primary and Metastatic Lung Tumors. Cancer Res Treat. 2014;46(4):403-410.

[4] International Commission on Radiation Units and Measurements Report 62: Prescribing, Recording, and Reporting Photon Beam Therapy (Supplement to ICRU Report 50) Bethesda, MD: ICRU; 1999.

[5] Rosenzweig KE, Hanley J, Mah D, et al.The deep inspiration breath-hold technique in the treatment of inoperable non-small-cell lung cancer. Int J Radiat Oncol Biol Phys.200;48(1):81-87.

[6] Radiation Therapy Oncology Group . 2014 Mar 6 - RTOG 0915 Protocol Information, A Randomized Phase II Study Comparing 2 Stereotactic Body Radiation Therapy (SBRT) Schedules for Medically Inoperable Patients with Stage I Peripheral Non-Small Cell Lung Cancer. Philadelphia: RTOG; Mar, 2011 https://www.rtog.org/ClinicalTrials/ProtocolTable/StudyDetails.aspx?study=0915

[7] International Commission on Radiation Units and Measurements. Prescribing, recording, and reporting photon-beam intensitymodulated radiation therapy (IMRT): contents (ICRU Report 83). J ICRU. 2010;10:NP.

[8] Josipovic M,. Persson GF, et al. Deep inspiration breath hold radiotherapy for locally advanced lung cancer: Comparison of different treatment techniques on target coverage, lung dose and treatment delivery time. Acta Oncol. 2013;52(7):1582-1586.

[9] Giraud P, Morvan E, Claude L, et al. Respiratory Gating Techniques for Optimization of Lung Cancer Radiotherapy. J Thorac Oncol. 2011;6(12): 2058-2068. 